\title{
Cardiac testing and outcomes in infants after an apparent life-threatening event
}

\author{
Robert Hoki, ${ }^{1}$ Joshua L Bonkowsky, ${ }^{2}$ L LuAnn Minich, ${ }^{1}$ Rajendu Srivastava, ${ }^{3}$ \\ Nelangi M Pinto ${ }^{1}$
}

\begin{abstract}
${ }^{1}$ Division of Pediatric
Cardiology, Department of Pediatrics, University of Utah School of Medicine, Salt Lake City, Utah, USA

'Division of Pediatric Neurology, Department of Pediatrics, University of Utah School of Medicine, Salt Lake City, Utah, USA

${ }^{3}$ Division of Inpatient Medicine, Department of Pediatrics, University of Utah School of Medicine, Salt Lake City, Utah, USA
\end{abstract}

\section{Correspondence to} Dr Nelangi M Pinto, Primary Children's Medical Center, Division of Pediatric Cardiology, Department of Pediatrics, University of Utah School of Medicine, 100 N. Mario Capecchi Drive, Salt Lake City, UT 84058, USA; nelangi. pinto@imail.org

Accepted 31 August 2012 Published Online First 25 September 2012

\begin{abstract}
Objectives We sought to determine the yield of cardiac testing and to identify predictors of cardiac disease in infants with an apparent life-threatening event (ALTE).

Design Retrospective longitudinal cohort study.

Setting Paediatric hospital providing primary and tertiary care that is part of an integrated healthcare system.

Patients Infants hospitalised for an ALTE from 1999 to 2003

Main exposures Cardiac testing used at time of ALTE and results, and clinical risk factors for cardiac disease.
\end{abstract}

Outcome measures Short-term (during hospitalisation) and long-term (through November 2009) follow-up for any diagnosis of significant cardiac anatomic or rhythm abnormality.

Results Study criteria were met by 485 infants (mean age 1.9, SD \pm 2.2 months; $49 \%$ boys). Cardiac testing was performed on 219 (45\%) patients during ALTE hospitalisation, identifying two patients with significant cardiac disease (cardiomyopathy, ventricular preexcitation). During 7.7 years of follow-up, three additional significant cardiac diagnoses (ventricular pre-excitation, frequent ventricular ectopy, moderate aortic stenosis) were identified. All cardiac tests had low positive predictive value (PPV). Significant cardiac disease was associated with prematurity (22\% vs $80 \%, p=0.002)$, but not age, gender, prior ALTE or rescue breaths.

Conclusions This longitudinal study of an ALTE cohort revealed significant cardiac disease in $<1 \%$ of patients. Prematurity was the only clinical predictor identified. ECG was sensitive for identifying significant cardiac disease, but routine testing warrants further investigation because of the low PPV.

\section{INTRODUCTION}

Apparent life-threatening events (ALTEs) present a diagnostic challenge. The definition of ALTE ('an episode that is frightening to the observer, characterised by some combination of apnoea (central or obstructive), colour change (cyanotic, pallid, erythematous, or plethoric), change in muscle tone (usually diminished), choking, or gagging') ${ }^{1}$ is ambiguous. The differential diagnoses include diseases that affect the gastrointestinal, respiratory, neurological and cardiovascular systems. Despite all these potential aetiologies, the cause of an ALTE remains unclear in $23-50 \%$ of cases, ${ }^{2-4} \mathrm{com}-$ plicating management decisions.

Well-appearing infants evaluated for an ALTE have a low mortality risk, ${ }^{5}$ and a significant association with an eventual diagnosis of epilepsy and abuse. ${ }^{67}$ Cardiac aetiologies and outcomes are not well-characterised. It is estimated roughly $<5 \%$ of

\section{What is already known on this topic}

- Cardiac diagnoses as an initial finding at the time of an apparent life-threatening event (ALTE) are rare.

- There are no standards for cardiac testing in infants who present with an ALTE.

- Prematurity has an increased prevalence in patients who present with an ALTE.

\section{What this study adds}

- The prevalence of cardiac disease remained low in this population of patients who were followed for several years beyond the time of presentation with an ALTE.

- Cardiac testing done at the time of an ALTE has a low predictive value for diagnosing cardiac disease.

- Prematurity may be an independent risk factor for the presence of cardiac disease in patients who present with an ALTE.

ALTEs have a cardiac aetiology, ${ }^{2}$ but the lack of long-term follow-up studies makes the true prevalence of cardiac disease unknown. A potential cardiac diagnosis for an ALTE carries a disparate amount of anxiety for families and clinicians concerned about the potential need for surgery and the risk of sudden death. There are no widely accepted recommendations for cardiac testing and the yield of testing in infants with ALTE remains unknown.

We sought to determine the prevalence of undiagnosed heart disease (significant cardiac anomalies or arrhythmias), to ascertain the frequency and yield of cardiac testing, and to identify predictors for cardiac disease in infants who present with an ALTE without an obvious diagnosis at presentation.

\section{METHODS}

\section{Design}

We performed a retrospective chart review of infants $<12$ months of age presenting to Primary Children's Medical Center (PCMC, Salt Lake City, Utah, USA) for an ALTE between January 1999 and December 2003. PCMC is the primary tertiary paediatric referral centre for Utah and four other Rocky Mountain states and is in the integrated Intermountain Healthcare (IHC) system that 
includes 23 hospitals in Utah and Idaho. To identify all patients admitted with an ALTE, the hospital databases were electronically searched for keywords in the chief complaint at presentation to the emergency department, and/or for the International Classification of Diseases, 9th Revision, Diagnostic Manual (ICD-9 DM) codes from consultations and inpatient discharge diagnoses. This was necessary to identify the most complete representation of patients with ALTE as possible since there were no ICD-9 codes specific to ALTE at the time of the study. The previously described keywords search included a wide variety of respiratory, neurological and gastrointestinal complaints. ${ }^{5}$ Charts were reviewed manually to include infants $\leq 12$ months of age at the time of presentation with a history consistent with ALTE. This was defined as a sudden event where the caregiver was concerned with one or more of the following: breathing irregularity (apnoea, choking, gagging), colour change (cyanosis, pallor), altered muscle tone (hyoptonia or hypertonia), abnormal movements or altered mental status. Infants with a previous diagnosis that likely explained the ALTE, such as cardiac disease, severe gastroesophageal reflux (if presentation was consistent), genetic or metabolic disorders with central nervous system involvement, or a history of seizures or central nervous system pathology were excluded. Also excluded were infants whose ALTE could be reasonably attributed to an apparent diagnosis on presentation such as an obvious arrhythmia, respiratory tract infection, drug overdose, trauma, febrile seizure or child abuse. ${ }^{5}$ Patients with unstable or critical vital signs were not excluded since this can be consistent with significant cardiac disease.

We followed the cohort through November 2009 to identify cardiac disease diagnosed by subsequent evaluation unrelated to their initial ALTE. All patients presenting to PCMC or any of the 23 hospitals or affiliated clinics in the IHC system, which cares for a significant majority of children in Utah, were captured in follow-up. IHC maintains integrated electronic records (including admissions, emergency department visits, paediatric cardiology clinic visits, ECG and echocardiogram results), allowing comprehensive tracking of subsequent outcomes. We were also able to track patients referred to paediatric cardiology as PCMC is the only paediatric cardiology practice in Utah.

Deaths were identified through IHC's Enterprise Data Warehouse and through state health death records and were verified by examination of the patient medical record. Finally, we queried national death records (Social Security Database Index) by name and social security number. ${ }^{9}{ }^{10}$ Most recent follow-up for deaths was through June 2010.

\section{Data collection}

PCMC underwent a transition from paper to electronic medical records during the study period. We searched both sources to identify infants with cardiac testing at presentation or during subsequent evaluation in the follow-up period. Cardiac testing included ECG, echocardiography, formal rhythm monitoring (eg, Holter monitors and event recorders) and cardiology consultation. If cardiac tests were ordered as part of the initial ALTE evaluation but performed afterwards, they were considered to have occurred at presentation. We obtained demographic data, medical history and potential risk factors, including age at presentation, gender, prematurity ( $<37$ weeks' gestation), history of prior ALTE, use of rescue breaths, family history (sudden death, sudden infant death syndrome (SIDS) or cardiac disease) and abnormal clinical cardiac exam (abnormal rhythm, murmur, hepatomegaly or concerning chest x-ray).

\section{Cardiac testing and study definitions}

Test results were taken from study reports. All echocardiograms, Holter/event monitors and ECGs were read by a paediatric cardiologist specialising in these areas. In addition to major abnormalities, ECGs with possible ventricular hypertrophy, borderline interval lengths (including OTc), complete bundle branch block and first-degree heart block based on agedefined normals were considered abnormal. Ventricular conduction delay, early repolarisation pattern and isolated single premature atrial or ventricular complexes were considered normal. On echocardiography, isolated right aortic arch, left superior vena cava or patent foramen ovale were considered to be normal variants. 'Significant cardiac disease' was defined as congenital or acquired heart defect excluding isolated minor valve abnormalities, small septal defects or shunts and mild ventricular dysfunction; or arrhythmia diagnosis excluding isolated ectopy (ventricular or atrial).

\section{Analysis}

Analyses were conducted using Stata V.11.0. Descriptive statistics were determined for the study cohort. Continuous variables were examined for normality, and if met, were described as a mean with a SD. Fisher's exact test or Student's t test were used to determine univariate predictors of significant cardiac disease. Those with missing data for the variable of interest were not included in the analysis. The sensitivity, specificity, positive predictive value (PPV) and negative predictive value were calculated for the clinician's decision to obtain testing at the time of the ALTE, and the result of the tests obtained in relation to the determination of 'significant cardiac disease'. A clinician's decision to obtain a cardiac test was evaluated in relation to the presence of 'significant cardiac disease' regardless of the test result. This was examined to determine the relationship of the use of cardiac testing with the presence of cardiac disease. The characteristics of the test itself were based on whether the test was positive (abnormal result) in relation to the presence or absence of 'significant cardiac disease'.

The study was approved by the University of Utah and PCMC Institutional Review Boards.

\section{RESULTS}

\section{Patient selection}

After 187903 patient records were screened, 1148 infants had a presentation consistent with ALTE. Of these, seven were excluded for incomplete records, 180 were excluded for having a previous diagnosis that explained the ALTE, and 476 were excluded for one or more of the following: an acute condition that explained the ALTE, including bronchiolitis (176), seizure disorder (231), other central nervous system pathology (75), treatment with an automated external defibrillator (AED) (66), febrile seizures (33), medication overdose (13) and trauma (14). Only one patient was excluded for a prior diagnosis (congenital heart disease, tetralogy of Fallot). The mean age of 485 infants in the cohort was $1.9(\mathrm{SD} \pm 2.2)$ months (table 1). The mean length of follow-up after the ALTE was 7.7 ( $\mathrm{SD} \pm 1.3$ ) years. The most common presentation was choking or apnoea while awake in 194 patients (47\%). Several patients ( $n=121,29 \%$ ) were found blue or apneic with unclear precipitating circumstances. Abnormal movements with cyanosis were seen in $46(11 \%)$ and an additional $53(13 \%)$ presented with respiratory symptoms. Family history was only documented in $53 \%$ $(n=257)$ of cases. 
Table 1 Selected demographic and follow-up data on infants presenting with an ALTE

\begin{tabular}{|c|c|c|c|c|c|}
\hline Characteristic & $\begin{array}{l}\text { Cohort } \\
(n=485)\end{array}$ & $\begin{array}{l}\text { Missing } \\
\text { data }\end{array}$ & $\begin{array}{l}\text { Cardiac } \\
\text { testing at } \\
\text { time of ALTE } \\
(n=219)\end{array}$ & $\begin{array}{l}\text { No testing } \\
\text { at time of } \\
\text { ALTE } \\
(\mathrm{n}=266)\end{array}$ & $\begin{array}{l}p \\
\text { Value }\end{array}$ \\
\hline Boys, n (\%) & $240(50)$ & $0(0)$ & $109(50)$ & 131 (49) & 0.90 \\
\hline $\begin{array}{l}\text { Age in months, } \\
\text { mean (SD) }\end{array}$ & $1.9( \pm 2)$ & $2(0)$ & $2.1( \pm 2.4)$ & $1.8( \pm 2)$ & 0.15 \\
\hline $\begin{array}{l}\text { Premature } \\
(<37 \text { weeks), n } \\
(\%)\end{array}$ & $104(22)$ & $0(0)$ & $40(18)$ & $70(26)$ & 0.04 \\
\hline $\begin{array}{l}\text { History of prior } \\
\text { ALTE, } n(\%)\end{array}$ & $131(27)$ & $16(3)$ & $70(27)$ & $61(29)$ & 0.59 \\
\hline $\begin{array}{l}\text { Use of rescue } \\
\text { breaths, } n(\%)\end{array}$ & 43 (9) & $28(6)$ & $20(10)$ & $23(9)$ & 0.89 \\
\hline $\begin{array}{l}\text { Family history of } \\
\text { sudden death, } \\
\text { SIDS, or cardiac } \\
\text { disease, n (\%) }\end{array}$ & $12(5)$ & $230(47)$ & $9(8)$ & $3(2)$ & 0.04 \\
\hline $\begin{array}{l}\text { Clinical cardiac } \\
\text { indication, n (\%) }\end{array}$ & $29(6)$ & $0(0)$ & $20(4)$ & $19(7)$ & 0.50 \\
\hline $\begin{array}{l}\text { Follow-up years, } \\
\text { mean (SD) }\end{array}$ & $7.7( \pm 1)$ & $0(9)$ & & & \\
\hline
\end{tabular}

ALTE, apparent life-threatening event; SIDS, sudden infant death syndrome.

\section{Cardiac testing}

At presentation, 219 (45\%) infants underwent 254 cardiac tests and 31 cardiology consultations. Age and previous event were not related to the use of testing. Premature infants were less likely to have had cardiac testing at the time of their ALTE, while those who had a positive family history were more likely (table 1). ECG was most frequently used at ALTE (43\%, table 2). In follow-up, an additional 26 patients underwent cardiology testing for indications unrelated to the initial ALTE, with the most common indications being murmur and rhythm abnormalities.

\section{Testing results}

Overall, 20 of 485 patients (4\%) were identified with cardiac abnormalities (table 3). Of these, 15 were identified at ALTE presentation while 5 were identified subsequently. The majority of these infants had minor septal or valve defects. Only 5 of $485(1 \%)$ infants met the criteria for significant cardiac disease. Two were diagnosed at the ALTE (Wolff-Parkinson White

Table 2 CARDIAC testing in patients with ALTE

\begin{tabular}{|c|c|c|c|c|c|}
\hline Testing & $\begin{array}{l}\text { Evaluation } \\
\text { at ALTE }\end{array}$ & $\begin{array}{l}\text { Evaluation } \\
\text { after ALTE }\end{array}$ & $\begin{array}{l}\text { Evaluation } \\
\text { without } \\
\text { significant } \\
\text { cardiac } \\
\text { diagnosis } \\
(\mathrm{n}=480)\end{array}$ & $\begin{array}{l}\text { Evaluation } \\
\text { with } \\
\text { significant } \\
\text { cardiac } \\
\text { diagnosis } \\
(\mathrm{n}=5)\end{array}$ & $\begin{array}{l}\text { p } \\
\text { Value* }\end{array}$ \\
\hline $\begin{array}{l}\text { Any form of } \\
\text { cardiac testing }\end{array}$ & $216(45)$ & $26(5)$ & $213(44)$ & $3(60)$ & 0.66 \\
\hline $\begin{array}{l}\text { Cardiology } \\
\text { consult }\end{array}$ & $31(6)$ & $23(5)$ & $29(6)$ & $2(40)$ & 0.04 \\
\hline ECG & $208(43)$ & $25(5)$ & $205(43)$ & $3(60)$ & 0.66 \\
\hline Echocardiogram & $40(8)$ & $11(2)$ & $38(8)$ & $2(40)$ & 0.06 \\
\hline Rhythm testing & $6(1)$ & $9(3)$ & $5(1)$ & $1(20)$ & 0.06 \\
\hline
\end{tabular}

Data presented as $n(\%)$.

${ }^{*}$ p Value is comparison of those evaluated without a significant cardiac diagnosis to those evaluated with a significant cardiac diagnosis.

ALTE, apparent life threatening event.
Table 3 Cardiac diagnoses in patients who presented with an ALTE

Diagnoses at the time of ALTE

Wolff-Parkinson-White syndrome

Dilated cardiomyopathy

Small VSD (4)

Small PDA (3)

Bicuspid aortic valve (2)

Atrial septal defect (2)

Mild pulmonary stenosis

Right ventricular hypertrophy

Diagnoses after ALTE

Wolff-Parkinson-White syndrome

Frequent ventricular ectopy

Moderate aortic stenosis

Small VSD

Small ASD

If more than one patient, (n) is in parentheses. Significant cardiac diseases are shown in italics.

ALTE, apparent life-threatening event; ASD, atrial septal defect; PDA, patent ductus artery; VSD, ventricular septal defect.

syndrome (WPW) and idiopathic dilated cardiomyopathy with prominent right ventricle involvement). WPW was diagnosed as an incidental finding from a screening ECG without concern for previous tachycardia. The patient with cardiomyopathy had an enlarged liver which directed further testing. In the follow-up period, three additional patients were diagnosed with significant cardiac disease (WPW, frequent ventricular ectopy and moderate aortic stenosis) by subsequent testing done for reasons unrelated to the ALTE presentation.

The decision of physicians to obtain cardiac testing had a sensitivity of $60 \%$ for identifying patients who ultimately turned out to have significant cardiac disease (table 4). The sensitivity of an abnormal ECG obtained at the time of the ALTE was high $(100 \%)$ for identifying patients with significant cardiac disease compared with that of an abnormal echocardiogram (50\%, 1 out of 2 who had an echo at the ALTE, table 4). Despite the low prevalence of cardiac disease, a positive (abnormal) test result occurred in 97 of 214 (45\%) infants with cardiac testing at the ALTE presentation, including 94 of 207 (45\%) ECGs and 11 of 40 (28\%) echocardiograms. ECG abnormalities included 11 patients with mildly prolonged OTc, one of whom was the patient with cardiomyopathy. Of the remaining 10 patients, 6 had a normal repeat ECG or Holter, while 4 had no follow-up testing. Of these, three had mild prolongations ( $<450 \mathrm{~ms}$ ), but one had a OTc of $470 \mathrm{~ms}$ at 3 days of age. This patient's last documented visit was in 2010, with no follow-up ECGs in the IHC system and no documented events. No patient in this cohort had an established diagnosis of prolonged QT.

There were two deaths in the cohort during the follow-up period. Both were related to complications from chronic neurologic conditions that developed after the index ALTE hospitalisation. No adverse cardiac events were documented in follow-up.

\section{Predictors of cardiac disease}

Following univariate analysis, only prematurity predicted significant cardiac disease in the cohort (table 5). Of five infants with significant cardiac disease, four were premature. However, premature infants were less likely to have a cardiac evaluation at the time of their ALTE (table 1). No significant association was found for age, gender, history of prior ALTE, use of rescue breaths or positive family history with either significant or insignificant cardiac disease. While cardiac indications were not related to significant cardiac disease, they were positively associated with the presence of minor cardiac diagnoses. 
Table 4 Sensitivity, specificity and predictive values of the physician determining appropriate testing at the time of ALTE and for cardiac test results for significant cardiac disease $(n=5)$

\begin{tabular}{|c|c|c|c|c|}
\hline & Sensitivity (95\% CI) & Specificity (95\% CI) & PPV (95\% Cl) & NPV (95\% CI) \\
\hline Cardiology consultation* $(n=30)$ & 40 (7 to 83$)$ & 94 (91 to 96$)$ & $6(1$ to 23$)$ & $99(98$ to 100$)$ \\
\hline Echocardiogram obtained ${ }^{*}(n=40)$ & 40 (7 to 83$)$ & 92 (89 to 94$)$ & $5(1$ to 18$)$ & 99 (98 to 100$)$ \\
\hline ECG obtained ${ }^{*}(n=207)$ & 60 (17 to 93$)$ & 57 (53 to 62$)$ & $1(0$ to 4$)$ & 99 (97 to 100$)$ \\
\hline Any testing obtained ${ }^{*} \ddagger(n=214)$ & 60 (20 to 92$)$ & 55 (50 to 59$)$ & $1(0$ to 4$)$ & 99 (97 to 100$)$ \\
\hline Abnormal echocardiogram $^{\dagger}(n=11)$ & 50 (3 to 97$)$ & 74 (57 to 86$)$ & $9(0$ to 43$)$ & $97(80$ to 100$)$ \\
\hline Abnormal ECG† $(n=94)$ & $100(31$ to 100$)$ & 55 (48 to 62 ) & $3(1$ to 10$)$ & 100 (96 to 100$)$ \\
\hline Abnormal Holtert $(n=1)$ & $100(5$ to 100$)$ & $100(46$ to 100$)$ & $100(5$ to 100$)$ & $100(46$ to 100$)$ \\
\hline
\end{tabular}

*Physician decision to obtain test.
†Test result.
$\ddagger$ Any screening measure indicates patients who received at least one form of cardiac testing. Some patients received more than one test.
NPV, negative predictive value; PPV, positive predictive value.

\section{DISCUSSION}

Our study confirms the prevalence of cardiac disease in patients who present with an ALTE with an unclear diagnosis at presentation is very low, even after comprehensive longitudinal follow-up, resulting in a low yield for cardiac testing. Though performed in almost half of patients, use of cardiac testing was inconsistent. This is likely because identifiable predictors are few and only prematurity was associated with the presence of significant cardiac disease.

Our study demonstrated significant cardiac disease in $1 \%$ of patients with ALTE and minor cardiac disease in $4 \%$. This result is similar to that reported previously in ALTE and the general population. ${ }^{211}$ However, we were able to longitudinally follow patients for on average almost 8 years after the ALTE. Therefore, we can be reasonably certain that our results capture clinically significant cardiac disease. Although studies have analysed the aetiology of ALTEs, including the cardiac causes, ${ }^{4} 12$ this is the first study to follow a large cohort of patients and compare the use of cardiac testing and results at ALTE presentation with longitudinal cardiac outcomes. Although cardiac testing was done frequently, overall, its use seems to rely on individual physician practice. While having a family history of cardiac disease, sudden death or SIDS was associated with the use of cardiac testing, two patients with a positive family history had no cardiac testing. The presence of a documented clinical indication was rare and was not associated with the use of testing. Additionally, two-thirds of patients who were diagnosed subsequently with significant cardiac disease had no cardiac testing at the time of their ALTE. However, it is possible

Table 5 Risk factors for significant cardiac disease in infants presenting with an ALTE

\begin{tabular}{lcll}
\hline & $\begin{array}{l}\text { Without significant } \\
\text { cardiac disease, } \\
\mathbf{n = 4 8 0}\end{array}$ & $\begin{array}{l}\text { With significant } \\
\text { cardiac disease, } \\
\mathbf{n = 5}\end{array}$ & $\begin{array}{l}\mathbf{p} \\
\text { Value }\end{array}$ \\
\hline Mean age, months (SD) & $2.0( \pm 2.2)$ & $1.7( \pm 1.7)$ & 0.79 \\
Boys & $238(50)$ & $2(40)$ & 0.67 \\
Non-white race & $82(18)$ & $1(20)$ & 0.90 \\
Prematurity (<37 weeks) & $106(22)$ & $4(80)$ & 0.002 \\
History of prior event & $131(28)$ & $0(0)$ & 0.16 \\
Need for rescue breaths & $43(9)$ & $0(0)$ & 0.71 \\
Family history of sudden & $12(5)$ & $0(0)$ & 1.0 \\
death, SIDS or cardiac & & & 0.27 \\
disease & $29(6)$ & $0(0)$ & \\
Clinical cardiac indication & 29 &
\end{tabular}

Data are presented as $n(\%)$ unless indicated otherwise.

ALTE, apparent life threatening event; SIDS, sudden infant death syndrome. that there were other factors that are not readily apparent by documentation that led to these decisions.

An ECG is a quick, non-invasive, and relatively inexpensive test, often obtained in an effort to 'rule out' cardiac causes. Indeed, ECG was sensitive for identifying cardiac disease in our cohort of patients. Of five patients with significant cardiac disease, three had a primary rhythm disturbance diagnosed by ECG abnormalities. The ECG was also abnormal for the two patients with structural heart disease (cardiomyopathy and moderate aortic stenosis). While the predictive value of all cardiac tests was low due to the low prevalence of disease, the rate of false-positive results was high, especially for abnormal ECG findings (45\%). Many ECG abnormalities were minor, transient, and of little or unknown significance. Prior studies have also demonstrated ECG abnormalities to be common in patients who present with an ALTE and in healthy newborns, limiting its usefulness for cardiac diagnosis. ${ }^{12-14}$ Mild prolongation of the QT interval, while certainly not minor, is difficult to use for diagnosis in infants under 6 months of age during which time this interval fluctuates. ${ }^{15}$ No patient in our cohort was ultimately diagnosed with long QT, although four patients had no follow-up. Given the high percentage of false-positive results, testing may sometimes place psychological and financial burden on the family and healthcare providers, although the psychological burden in particular may be low in comparison to the general anxiety related to having an unknown aetiology for the child's ALTE. In addition, the possibility of detecting clinically significant disease and ECG sensitivity for screening for significant disease may outweigh the disadvantages of false-positive results.

The echocardiogram was a poor choice in patients who present with an ALTE. The utility of echocardiography as an initial test for ALTE appears limited in the absence of clinical findings on examination or ECG abnormalities and should likely be considered only after consultation with a cardiologist.

Studies have shown an increased prevalence of prematurity in patients who present with an ALTE. ${ }^{16} 17$ Prematurity was the only independent risk factor identified for significant cardiac disease in this study. However, premature infants were less likely to receive cardiac testing in this study. This may be because premature infants were thought to have other likely aetiologies for an ALTE (apnoea of prematurity, respiratory disease or reflux), although our ability to determine factors that impacted these clinical decisions was limited. While prematurity may increase suspicion for a cardiac aetiology of an ALTE, targeted testing in this population will need to be balanced with the potential for false-positive results. Additionally, the fact that there were no other identifiable risk factors, such as 
family history or documented clinical indications with cardiac disease, highlights the difficulty in trying to use selective cardiac testing in this population.

\section{LIMITATIONS}

There are several limitations to this study. The cohort represented a single centre whose patient demographic may not be generalisable. The search for patients who presented with an ALTE, while meant to be exhaustive, was dependent on the accuracy of the chief complaint in the emergency room, discharge diagnoses in hospital records and our study definition of ALTE. Additionally, exclusion of a previously diagnosed seizure disorder may have inadvertently excluded patients with an arrhythmia-induced seizure. However, we made the assumption that this possibility had been considered and ruled out. This study focused on patients with ALTE who were well and had an unclear diagnosis at presentation. This excluded patients who had an obvious arrhythmia or arrest requiring an AED at presentation, a patient group in which further cardiac testing is warranted. The retrospective nature of this study limited our ability to understand factors that led to physician decision-making that were not documented. A family history of a sudden event or arrhythmia may lead a clinician to use testing very differently, but this documentation was also inconsistent. It is possible that asymptomatic patients with heart disease may not have presented during the follow-up period and follow-up was limited to encounters with the IHC system. Finally, what defines significant cardiac disease is controversial and while a cardiac cause of ALTE would be a more useful outcome, this required too many assumptions for a retrospective study such as ours.

\section{CONCLUSIONS}

The majority of ALTE in young infants are not of cardiac origin. This low prevalence brings into question the value of non-selective or routine cardiac screening for young infants after an ALTE. However, though prematurity was a risk factor for significant cardiac disease, there are no other clear factors to guide cardiac testing. If cardiac screening is undertaken, ECG is sensitive for significant arrhythmogenic and structural heart disease in patients with ALTE, although the specificity and PPV are low. Thus further study of other factors that may guide testing decisions and the cost effectiveness of an ECG screen in the setting of ALTE is warranted.
Acknowledgements This study was supported by a NICHD grant for Dr Srivastava (K23 HD052553), a NIDA grant for Dr Bonkowsky (K08 DA24753), and the Children's Health Research Center, University of Utah and a NCI grant for Dr Pinto (1KM1CA156723-01).

Contributors Specifically, RH, NMP, JLB, RS and LLM were all involved in the conception and design of the study. RH and NMP were involved in the analysis and interpretation of the data. All authors were involved in drafting and revising the manuscript and have given their final approval for it in its current form.

\section{Competing interests None.}

Ethics approval University of Utah and Primary Children's Institutional Review Board.

Provenance and peer review Not commissioned; externally peer reviewed.

\section{REFERENCES}

1. National Institute of Health. Infantile apnea and home monitoring. Nat/ Inst Health Consens Dev Conf Consens Statement 1986;6:1-10.

2. McGovern MC, Smith MB. Causes of apparent life threatening events in infants: a systematic review. Arch Dis Child 2004:89:1043-8.

3. Davies F, Gupta R. Apparent life threatening events in infants presenting to an emergency department. Emerg Med J 2002;19:11-16.

4. Hall KL, Zalman B. Evaluation and management of apparent life-threatening events in children. Am Fam Physician 2005;71:2301-8.

5. Bonkowsky JL, Guenther E, Filloux FM, et al. Death, child abuse, and adverse neurological outcome of infants after an apparent life-threatening event. Pediatrics 2008;122:125-31.

6. Bonkowsky JL, Guenther E, Srivastava R, et al. Seizures in children following an apparent life-threatening event. J Child Neurol 2009;24:709-13.

7. Guenther $\mathbf{E}$, Powers A, Srivastava R, et al. Abusive head trauma in children presenting with an apparent life-threatening event. J Pediatr 2010;157:821-5.

8. Dewolfe CC. Apparent life-threatening event: a review. Pediatr Clin North Am 2005;52:1127-46.

9. Hill ME. The Social Security Agency's Death Master File: the completeness of death reporting at older ages. Soc Secur Bull 2002;65:45-51.

10. Genealogy Bank. Search the Social Security Death Index. Secondary Search Social Security Death Index. http://www.genealogybank.com/gbnk/ssdi/? $\mathrm{kbid}=9064 \mathrm{qm}=9$ (accessed 11 september 2012)

11. Hoffman JI, Kaplan S. The incidence of congenital heart disease. J Am Coll Cardio 2002;39:1890-900

12. Goldhammer El, Zaid G, Tal V, et al. OT dispersion in infants with apparent life-threatening events syndrome. Pediatr Cardiol 2002;23:605-7.

13. Woolf PK, Gewitz MH, Preminger T, et al. Infants with apparent life threatening events. Cardiac rhythm and conduction. Clin Pediatr (Phila) 1989;28:517-20.

14. Makarov LM, Komoliatova VN, Zeval'd SV, et al. Holter monitoring in healthy children during first days of life. Kardiologiia 2009;49:27-30.

15. Schwartz PS, Garson A, Paul T, et al. Guidelines for the interpretation of the neonatal electrocardiogram. Eur Heart J 2002;23:1329-44.

16. Weiss K, Fattal-Valevski A, Reif $S$. How to evaluate the child presenting with an apparent life-threatening event? Isr Med Assoc J 2010;12:154-7.

17. De Piero AD, Teach SJ, Chamberlain JM. ED evaluation of infants after an apparent life-threatening event. Am J Emerg Med 2004;22:83-6. 